\title{
Apoptosis in Young Rats with Adriamycin-Induced Cardiomyopathy- Comparison with Pirarubicin, a New Anthracycline Derivative
}

\author{
EIKAN KOH, YOSIMICHI UEDA, TSUNEYUKI NAKAMURA, AZUSA KOBAYASHI, \\ SHOGO KATSUTA, AND HIROAKI TAKAHASHI
}

Department of Pediatrics [E.K., T.N., A.K., H.T.], Department of Pathology [Y.U., S.K.], Kanazawa

Medical University, Kanazawa, 920-0293, Japan

\begin{abstract}
ABS
In a previous study, we demonstrated that apoptosis in rats
with adriamycin (ADR)-induced cardiomyopathy occurred
through a Fas-dependent pathway. Pirarubicin, a new anthracy-
cline derivative, seems to have a lower cardiotoxicity than ADR.
To investigate whether pirarubicin has a lower chronic cardio-
toxicity compared with ADR, ADR or pirarubicin were injected
weekly for 8 wk into young Wister rats via the tail vein. To block
the Fas-Fas ligand interaction, an anti-Fas ligand antibody (FasL)
was injected with ADR 7, 8, and 9 wk after first administration
of ADR. In the control group, saline was injected instead of
ADR. ADR significantly induced apoptosis and left ventricular
dysfunction 10 wk after the first administration of ADR. Piraru-
bicin also induced apoptosis, however, its apoptosis was signif-
\end{abstract}
Doxorubicin (ADR) is one of the most effective and useful antineoplastic agents for the treatment of hematological and solid malignancies. However, its practical therapeutic use is sometimes limited by late-onset and chronic cardiotoxicity (1, 2). A new anthracycline derivative, 4'-O-tetrahydropyranydal (THP)-adriamycin (pirarubicin; Meiji Seika, Tokyo, Japan, and Nihonkayaku, Tokyo, Japan) was introduced in 1979 (3). Compared with ADR, pirarubicin has a similarly high antitumor efficacy (4-6) and a much lower acute cardiotoxicity (7, $8)$. However, it is unclear whether the chronic cardiotoxicity of pirarubicin is lower than that of ADR. In our earlier study, we observed in rats with ADR-CM that apoptosis occurred through a Fas-dependent pathway (9). Our ADR-CM model in rat showed histologic changes similar to those in human ADR$\mathrm{CM}$ - degeneration and loss of myocytes, compensatory hypertrophy of residual myocytes, and interstitial fibrosis - and

Received May 1, 2001; accepted July 23, 2001.

Correspondence and reprint requests: Eikan Koh, Department of Pediatrics, Kanazawa Medical University, 1-1 Daigaku, Uchinada-machi, Kahoku-gun, Ishikawa, 920-0293, Japan; e-mail: p-koh@kanazawa-med.ac.jp

Supported by Grants-in-Aid from the Ministry of Education, Science, and Culture of Japan. icantly ( $p=0.0069)$ less than that induced by ADR. Fas antigen was overexpressed in the hearts of ADR and ADR + FasL groups, however, an expression of Fas antigen in the pirarubicin group was similar to the expression of Fas antigen in the control group. Thus, pirarubicin has a significantly lower chronic cardiotoxicity compared with ADR. (Pediatr Res 51: 256-259, 2002)
ADR, adriamycin
ADR-CM, adriamycin-induced cardiomyopathy
FasL, anti-Fas ligand antibody
$\mathbf{L V}$, left ventricle

Abbreviations showed an increase in the number of apoptotic myocardial cells with the progression of ADR-CM both histologically and functionally. In this study, we investigated in rat ADR-CM models, histologically, molecular biologically, and functionally, whether piracubicin has a lower chronic cardiotoxicity compared with ADR.

\section{MATERIALS AND METHODS}

Experimental protocol. ADR-CM was induced in six young male Wister rats weighing $59 \pm 5 \mathrm{~g}$ by weekly administration of $2 \mathrm{mg} / \mathrm{kg}$ of ADR (obtained from Kyowa Hakko, Japan) into tail veins for $8 \mathrm{wk}$, in accordance with the method of Podesta et al. (10). This animal study was approved by the Institutional Animal Care and Use Committee of Kanazawa Medical University. In three of the rats, to block Fas/Fas ligand interaction, $10 \mu \mathrm{g} / \mathrm{kg}$ of FasL (BD PharMingen, San Diego, CA, U.S.A.) was injected with ADR three times via the tail vein 6,7 , and 8 wk after the first administration of ADR (ADR + FasL group). Pirarubicin $2 \mathrm{mg} / \mathrm{kg}$ (obtained from Meiji Seika, Japan) was injected into three rats (pirarubicin group) by the same protocol and physiologic saline was injected into three control rats 
(control group). Echocardiograms of each rat, anesthetized with ether, were taken $6,7,8,9$, and $10 \mathrm{wk}$ after the first injections. After the last echocardiography, all the rats were killed, the chest was opened, and the heart was immediately isolated. The blood in the heart was removed, and the left ventricle was cut horizontally into three slices. The mid-slice of the left ventricle was fixed in $4 \%$ paraformaldehyde at $4^{\circ} \mathrm{C}$ for $8 \mathrm{~h}$, dehydrated in a graded alcohol series, and then embedded in paraffin. The apical and basal portions of the left ventricle were rapidly frozen in liquid nitrogen and were preserved at $80^{\circ} \mathrm{C}$ until use. A part of the left ventricle was also embedded in ornithine carbamoyltransferase compound and rapidly frozen.

Histologic assessment. Paraffin sections, $3 \mu \mathrm{m}$ thick, were stained with hematoxylin and eosin, reticulin silver impregnation, and Azan-Mallory stain.

Analysis of left ventricular performance. LV dimensions [the end-diastolic diameter (LVDd), end-systolic diameter (LVDs), the intraventricular septal thickness, and the LV posterior wall thickness] were measured by echocardiography using a SONOS 5500 with a $12-\mathrm{MHz}$ transducer (Agilent Technologies). The percent fractional shortening $(\% \mathrm{FS})$ of the $\mathrm{LV}$ was calculated from the following formula: $\% \mathrm{FS}=$ [(LVDd - LVDs $) /$ LVDd] $\times 100$.

In situ terminal deoxynucleotidyl transferase assay (TUNEL method). The TUNEL method is based on the specific binding of terminal deoxynucleotidyl transferase (TdT) to the 3 '-OH ends of DNA and the ensuing synthesis of a polydeoxynucleotide polymer. The TUNEL method was applied to $4 \%$ paraform-aldehyde-fixed, paraffin-embedded sections $3 \mu \mathrm{m}$ thick with an ApoTag In Apoptosis Detection Kit (Oncor, Gaithersburg, MD, U.S.A.) according to the manufacturer's instructions.

Quantification of apoptotic cells. Myocardial nuclei of 3 $\mu \mathrm{m}$ horizontal sections of the LV and the intraventricular septum were labeled by the TUNEL method. The nuclei were counted, and the apoptotic index (number of myocardial nuclei labeled by the TUNEL method/number of total myocardial nuclei) was calculated.

Western blot analysis. Proteins were extracted from freshly frozen LV myocardium. Homogenized myocardial tissue was lysed in a solution containing $50 \mathrm{mM}$ Tris- $\mathrm{HCl}(\mathrm{pH} 7.2), 50$ $\mathrm{mM} \mathrm{NaCl}, 1 \%$ Nonidet P-40, $1 \%$ sodium deoxycholic acid, and 2\% SDS with protease inhibitor cocktail (Roche Molecular Biochemicals, Mannheim, Germany). The protein concentration was evaluated against standard BSA with a Bio-Rad (Richmond, CA, U.S.A.) assay reagent. Twenty micrograms of each protein preparation was electrophoretically separated on a $15 \%$ SDS-PAGE gel and transferred to a nitrocellulose membrane. The membrane was incubated with anti-Fas antigen antibody (Transduction Laboratories, diluted at 1:2500) or anti- $\beta$ actin antibody (Sigma Chemical, St. Louis, MO, U.S.A.) diluted at 1:2000) for $1 \mathrm{~h}$ at room temperature. Western blots were exposed to $\mathrm{x}$-ray film with an enhanced chemiluminescence kit (Pierce Chemical, Indianapolis, IN, U.S.A.). Bands were identified by comparison with standards purchased from the same companies that supplied the antibody.

Data collection and analysis. All the results are presented as mean \pm SD. The statistical significances for comparisons of $\% \mathrm{FS}$ and apoptotic index between each of the four groups were determined with $t$ test. Values of $p<0.05$ were considered to be significant.

\section{RESULTS}

Ten weeks after the first injection, the body weights were $270 \pm 20 \mathrm{~g}$ in the ADR group, $262 \pm 4 \mathrm{~g}$ in the ADR + FasL group, $298 \pm 12 \mathrm{~g}$ in the pirarubicin group, and $336 \pm 5 \mathrm{~g}$ in the control group. Body weights of the ADR, ADR + FasL, and pirarubicin groups were significantly $(p=0.0454)$ lower than those of the control group and the body weights of the pirarubicin group were significantly higher than those of the ADR group.

$\boldsymbol{L} \boldsymbol{V}$ performance. The $\% \mathrm{FS}$ of LV diminished significantly in the ADR and ADR +FasL groups of rats compared with that of the control rats. The \% FS in the pirarubicin group was similar to that of the control group and significantly higher than that of the ADR group (Table 1).

Histopathology. Under the light microscope, in hematoxylin and eosin-stained sections of the ADR group, degeneration of myocardial tissue was seen: vacuolization of the cardiomyocytes, myofibrillar loss, myocardial hypertrophy, and interstitial fibrosis (Fig. 1). Pyknotic nuclei and fragmentation of the nuclei were sometimes found in the ADR group. The changes of the ADR+FasL and pirarubicin groups were milder than those of the ADR group.

Apoptotic cells. The TUNEL method labeled not only fragmented nuclei of the myocardial cells but also pyknotic nuclei (Fig. 1). Similar to a previous study, a small number of cells labeled by the TUNEL method was detected even in the control group (Table 2). Compared with the control group (0.12 \pm $0.04 \%$ ), the apoptotic index was significantly increased in the ADR group $(p=0.0036)$, in the ADR+FasL group ( $p=$ $0.0064)$, and in the pirarubicin group $(p=0.0165)$. However,

Table 1. Left ventricular performance in each group

\begin{tabular}{lccccc}
\hline & $\% \mathrm{FS}, \%$ & LVDd, mm & LVDs, mm & IVS, mm & LVPW, mm \\
\hline ADR group & $55.7 \pm 3.2^{*}$ & $6.8 \pm 0.1$ & $3.0 \pm 0.2$ & $1.3 \pm 0.1$ & $1.5 \pm 0.3$ \\
ADR + FasL group & $51.3 \pm 8.2^{* *}$ & $6.5 \pm 0.7$ & $3.1 \pm 0.2$ & $1.2 \pm 0.2$ & $1.6 \pm 0.2$ \\
Pirarubicin group & $65.6 \pm 5.3 \#$ & $6.6 \pm 0.3$ & $2.3 \pm 0.3$ & $1.5 \pm 0.1$ & $1.6 \pm 0.1$ \\
Control group & $64.3 \pm 3.7$ & $6.9 \pm 0.6$ & $2.5 \pm 0.5$ & $1.3 \pm 0.1$ & $1.6 \pm 0.3$ \\
\hline
\end{tabular}

Data are mean $\pm \mathrm{SD} ; \% \mathrm{FS}=[(\mathrm{LVDd}-\mathrm{LVDs}) / \mathrm{LVDd}] \times 100$; IVS, intraventricular septal thickness; LVPW, left ventricular posterior wall thickness.

* Significantly different from control $(p=0.0038)$.

** Significantly different from control $(p=0.042)$.

\# Significantly different from ADR group $(p=0.044)$. 


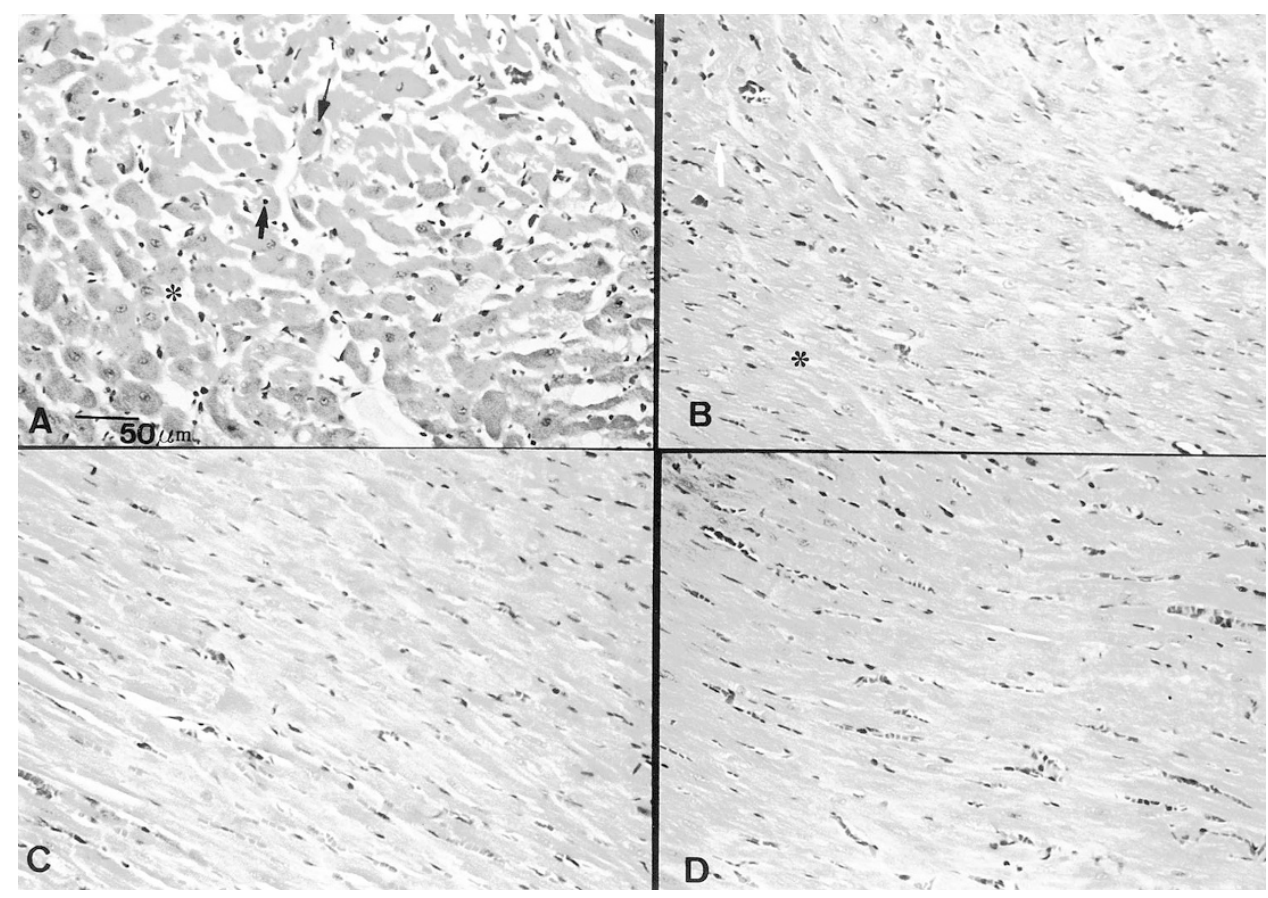

Figure 1. Light microscopic findings by hematoxylin-eosin-stained myocardium of rats injected with ADR $(A)$, ADR + FasL $(B)$, pirarubicin $(C)$, and saline $(D)$. Vacuolization of cardiomyocytes (shown by white arrows) and interstitial fibrosis (shown by an asterisk) are seen in $A$ and $B$. Black arrows indicate pyknotic and fragmented myocardial nuclei in $A$. Magnification: $A-D, \times 50$.

Table 2. Number of apoptotic cells in each group

\begin{tabular}{lcccc}
\hline & ADR group & ADR + FasL group & Pirabubicin group & Control group \\
\hline Number of apoptotic cells & $64 \pm 17$ & $29 \pm 10$ & $10 \pm 3$ & $7 \pm 2$ \\
Apoptotic index, \% & $1.29 \pm 0.33^{*}$ & $0.67 \pm 0.18^{* *}, \dagger^{* * * *}$ & $0.30 \pm 0.07 \#$ & $0.12 \pm 0.04$ \\
\hline
\end{tabular}

Apoptotic index, \%, indicates number of myocardial nuclei labeled by the TUNEL method/number of label of total myocardial nuclei. Data are mean \pm SD of three rats.

* Significantly different from control $(p=0.0036)$.

** Significantly different from control $(p=0.0064)$.

$\dagger^{* * *}$ Significantly different from control $(p=0.0165)$.

\# Significantly different from ADR group $(p=0.0069)$.

compared with the ADR group, the apoptotic index was significantly decreased in the pirarubicin group $(p=0.0069)$ and in the ADR +FasL group $(p=0.0468)$. The apoptotic index in the pirarubicin group was significantly lower than the index of the ADR +FasL group $(p=0.0273)$.

Expression of Fas antigen. Compared with the control group, Fas antigen was overexpressed in the heart of the ADR and ADR+FasL groups. However, the expression of Fas antigen in the pirarubicin group was similar to the expression of Fas antigen in the control group (Fig. 2).

\section{DISCUSSION}

This study demonstrated that pirarubicin had a lower chronic cardiotoxicity compared with ADR. ADR remains a major antitumor agent, despite it being well documented that the cardiotoxicity result in ADR-CM. The pathogenesis of ADR-CM remains unknown. However, we have demonstrated, in an in vivo study of ADR-CM in rats, the presence of apoptotic myocardial cells and the occurrence of the apoptosis through a Fas-dependent pathway. In that study, the apoptotic cells were detected by both the TUNEL method and by DNA agarose-gel electrophoresis, not only typical morphologic features of apoptosis (9). Based on the practicality and reliability of the TUNEL method in that study, we used in this study only the TUNEL method. We also have shown that with the accumulation of ADR, peaking at $10 \mathrm{wk}$ after the first administration of ADR, apoptotic myocardial cells increased, Fas antigen was overexpressed, and cardiac dysfunction deteriorated. In this study, the rats were killed and were examined $10 \mathrm{wk}$ after the first administration, because most rats with ADR-CM die as a result of heart failure $11 \mathrm{wk}$ after the first administration of ADR. Several recent reports have shown that myocardial cell death through apoptotic mechanism contributes to heart failure in a variety of cardiac diseases, including dilated cardiomyopathy and ischemic cardiomyopathy $(11,12)$. In this study, not only the histologic changes but also the number of apoptotic myocytes, the degree of overexpression of Fas antigen, and the serial LV performance were investigated to objectively compare chronic cardiotoxicities of pirarubicin and ADR.

Several experimental reports have shown that acute cardiotoxicity of pirarubicin is less than the cardiotoxicity of ADR (7, 8). However objective studies concerning chronic cardiotoxic- 


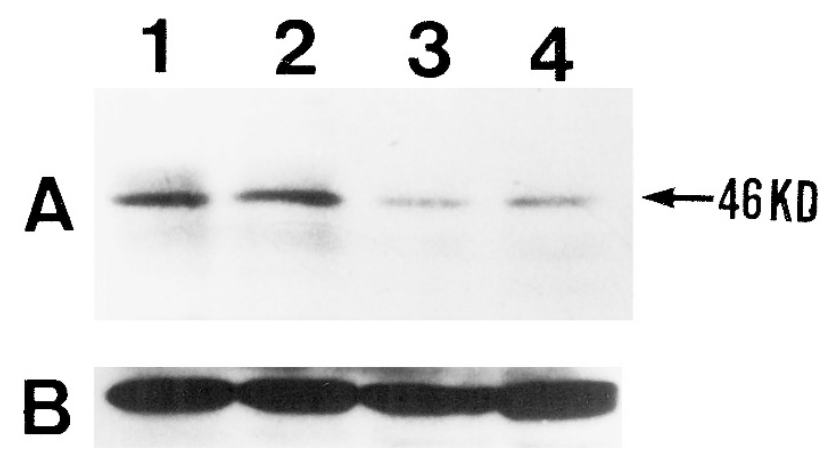

Figure 2. Western blots of the expression of Fas antigen $(A)$ and $\beta$-actin $(B)$. Lane 1 shows LV tissue of ADR-injected rat; lane 2, ADR+FasL-injected; lane 3, pirarubicin-injected; lane 4, saline-injected rat. Expression of Fas antigen is increased in ADR and ADR+FasL-injected heart tissue, but not significantly increased in pirarubicin-injected heart tissue.

ity of pirarubicin were not complete $(13,14)$. Compared with ADR-injected rats, pirarubicin-injected rats undergo significantly less histologic change of myocardial tissue, and have a smaller apoptotic index, less expression of Fas antigen, and no cardiac dysfunction. Furthermore, compared with ADR +FasL-injected rats, pirarubicin-injected rats have a smaller apoptotic index. Therefore, the neutralization of the anti-Fas ligand antibody is effective but seems to be limited. These findings objectively confirm that pirarubicin has a much lower chronic cardiotoxicity than does ADR. Based only on the result of the apoptotic index, the chronic cardiotoxicity of pirarubicin is quantitatively one-fourth of ADR.

It has been reported by Umezawa et al. (3) and Tsuruo et al. (6) that pirarubicin is a new anthracycline derivative and has antitumor activity against some experimental tumors. Furthermore, some clinical studies have shown that pirarubicin is effective against some cancers and that the substitution of pirarubicin for ADR preserved the high antitumor activity in some chemotherapeutic protocols $(13,15)$. On the other hand, ADR is one of the most effective and useful antitumor agents for the treatment of a variety of malignancies, including lymphomas, leukemia, and solid tumors. However, the therapeutic use of ADR should be abandoned because of its cardiotoxicity, especially late-onset and chronic cardiotoxicities (these are dose dependent), although its efficacy is still preserved. Several approaches have been tried to reduce these side effects $(16,17)$, however none of them have completely eliminated the cardiotoxicity. Furthermore, we have no effective and definitive treatment for anthracycline-induced cardiomyopathy other than heart transplantation.

Our results of an in vivo study show that the chronic cardiotoxicity of pirarubicin is significantly lower than the cardiotoxicity of ADR. Therefore, we recommend comparing the efficacy of pirarubicin and ADR in more clinical trials. It is hopeful that the substitution of ADR with pirarubicin will result in reduction in anthracycline-induced cardiomyopathy, and, furthermore, lead to a higher frequency of remission of many malignancies.

\section{REFERENCES}

1. Henderson IC, Frei E 1979 Adriamycin and the heart. N Engl J Med 300:310-312

2. Lefrak EA, Pitha J, Rosenheim S, Gottlieb JA 1973 A clinicopathologic analysis of adriamycin cardiotoxicity. Cancer 32:302-314

3. Umezawa $H$, Takahashi $Y$, Kinoshita $M$, Naganawa H, Masuda T, Ishizuka M, Tatsuta K, Takeuchi T 1979 Tetrahydropyranyl derivatives of daunomycin and adriamycin. J Antibiot 32:1082-1084

4. Hisamatsu T, Suzuki K, Sakakibara S, Komuro K, Nagasawa M, Takeuchi T, Umezawa H 1985 Tumor spectrum of a new anthracycline, (2"R)-4'-O-tetrahydropyranyladriamycin, and effect on the cellular immune response in mice. Jpn J Cancer Res 76:1008-1020

5. Matsushita Y, Kumagai H, Yoshimoto A, Tone H, Ishikura T 1985 Antitumor activities of (2"R)-4'-O-tetrahydropyranyl adriamycin (THP) and its combination with other antitumor agents on murine tumors. J Antibiot 38:1408-1419

6. Tsuruo T, Iida H, Tsukagoshi S, Sakurai Y 1982 47-O-tetrahydropyranyl-adriamycin as a potential new antitumor agent. Cancer Res 42:1462-1467

7. Dantchev D, Paintrand M, Hayat M, Mathe G 1979 Low heart and skin toxicity of a tetrahydropyranyl derivative of adriamycin (THP-ADM) as observed by electro- and light microscopy. J Antibiot 32:1085-1086

8. Kurebe M, Yokota M, Chesterman H, Massey JE, Gopinath C 1986 Toxicological studies on ( 2 "R)-47-O-tetrahydropyranyl-adriamycin, a new antitumor antibiotics. Its subacute toxicity and recovery study in beagle dogs. Jpn J Antibiot 39:351-401

9. Nakamura T, Ueda Y, Guan Y, Katsda S, Takahashi H, Koh E 2000 Fas-mediated apoptosis in adriamycin-induced cardiomyopathy in rats: in vivo study. Circulation 102:572-578

10. Podesta A, Della P, Pinciroli G, Iatropoulos MJ, Brughera M, Mazue G 1994 Evaluation of 4'-iodo-4'-deoxydoxorubicin-induced cardiotoxicity in two experimental rat models. Toxicol Pathol 22:68-71

11. Narula J, Haider N, Virmani R, DiSalvo T, Kolodgie FD, Hajjar RJ, Schmidt U, Semigran MJ, Dec GW, Khaw B 1996 Apoptosis in myocytes in end-stage heart failure. N Engl J Med 335:1182-1189

12. Olivetti G, Abbi R, Quaini F, Kajstura J, Cheng W, Nitahara JA, Quani C, Beltrami CA, Krajewski S, Reed JC, Anversa P 1997 Apoptosis in the failing human heart. N Engl J Med 336:1131-1141

13. Dhingra K, Frye D, Newman RA, Walters R, Theriault R Frschini G, Smith T, Buzdar A, Hortobagyi GN 1995 Phase II clinical and pharmacological study of pirarubicin in combination with 5-fluorouracil and cyclophosphamide in metastatic breast cancer. Clin Cancer Res 1:691-697

14. Suzuki M, Hirono M, Majima H 1997 Cardiotoxicity due to prolonged administration of THP (2"R-47-O-tetrahydropiranyl-adriamycin). Jpn J Cancer Chemother 24:19931999

15. Kaneko M, Tsuchida Y, Uchino J, Takeda T, Iwafuchi M, Ohnuma N, Mugishima H, Yokoyama J, Nishihira H, Nakada K, Sasaki S, Sawada T, Kawa K, Nagahara N, Suita S, Sawaguchi S 1999 Treatment results of advanced neuroblastoma with the first Japanese study group protocol. J Pediatr Hematol Oncol 21:190-197

16. Shapira J, Gotfried M, Lishner M, Ravid M 1990 Reduced cardiotoxicity of doxorubicin by a 6-hour infusion regimen. Cancer 65:870-873

17. Herman EH, Rahman A, Ferrans VJ, Viick JA, Schein PS 1983 Prevention of chronic doxorubicin cardiotoxicity in beagles by liposomal encapsulation. Cancer Res 43:5427-5432 